\title{
Concentraciones de aminoácidos en suero y orina en niños chilenos de 0-6 años de edad
}

\author{
Dr. Jorge Rodrigucz ${ }^{1}{ }^{1}$ : Q.F. Raquel Pino . $^{2}$ : Q.F. Edda Costa $C^{2}$ \\ Normal serum and urine aminoacid patterns in a chilean \\ population 0 to 6 years
}

\begin{abstract}
Inherited errors of aminoacid metabolism ase characterized by partial or total enzymatic deficiencies with accumulation of metabolites which reveal an altered metabolic pathway. The diagnosis of these inborn errors need: an available, jecurate and reliable laboratory technique. The ain of this study was to develop a gas-liculic chromatographic method for the analysis of aninoacids in biological fluids. This included three mairi stages: isolation and quantification of ammoacids. Normat serum and utine aminoacid patterns in a chileal population aged 0 to 6 ycars old are presented. Normal serum values showed targe range variations between traces (Tyrosin) and 1808 u molí (Onntin). Normal aminoaciduria also showed lurge variation according with age and the particular aminoacid in study.

(Key words: aminoacids, serum levels nomal childten).
\end{abstract}

El avance de la bioquímica clírica ha permitido una mejor comprensión de los procesos patológicos, aumentar la precisión en el diagnós. tico, despertar el interés por parte del clínico en las denomúnadas enfermedades congénitas del metabolisno y ampliar el conocimiento acerca de las enfermedades que afectan al metabolismo de los aminoácidos. Estas enfermedades presentan la caracteristica de ser hereditarias como consecuencia de mutación genética de cromosomas encargados de enzimas propias del metabolismo, to que determina un déficit enzimático parcial o total, produciendo la alteración de una via metabólica con el consecuente acumulo de un metabolito. ${ }^{1}$ Generalmente se manifjestan en la temprana infancia por un cuadro grave, en el cual el diagnóstico es dificil si no se dispone de pruebas bioquimicas especificas. En aquellos pacientes que no han sido diagnosticados y tratados en

1. Liscuela de Medicina, Universidad de Valparaíso.

2. Liscueta de Qúninica y Iarmacia, Universidad de Vatparaíso. forma oportuna, la muerte (forma clásica de la enfermedad olor jarabe de arce en orina) o el retardo mental (fenilcetonuria), se presentan con frecuencia.

El procedimiento de diagnóstico más exacto de una alteración metabólica es la determinación del déficit de la actividad enzimática. Esto requiere generalmente técnicas complejas $y$ de clevado costo.

En lo que se refiere a errores congénitos del metabolismo de aminoácidos, las técnicas de diagnóstico se han basado inicialmente en pruebas cualitativas para orina, diseñadas para determinar la concentración anormal de un metabolito, tales como pruebas del cloruro férrico, cianuro nitroprusiato, 2,4-dinitrofenillidrazina, prueba para la acidemia metilmalónica, entre otras. Sin embargo estas pruebas colorimetricas no son específicas, siendo sólo capaces de detertminar eliminaciones excesivas de algunos aminoácidos ${ }^{2}$. Un resultado positivo requiere necesariamente una investigación bioquimica más profunda que permita separar, identificar y cuantificar el metabolito excretado en forma anormal. 
Para superar estas dificultades se han empleado métodos bacteriológicos indirectos ${ }^{3}, a$ en forma más reciente métodos cromatográficos. Estos últimos pueden ser semicuantitativos en el caso de la cromatografía por partición ${ }^{4}$, o cuantitativos en el caso de la cromatografía en columnas de resinas de intercambio iónico ${ }^{5}$, cromatografía-liquido ${ }^{6,7}$ y HPLC.

En nuestro medio, desde el punto de vista de apoyo al diagnóstico, los errotes congénitos de metabolismo no se han enfocado en toda su nfagnitud, a través de técricas accesiblés y con. fiables, lo cual tiene obvias consecuencias en el tratamiento, pronóstico y consejo genético que dichos pacientes requieren. A nivel nacional, a nuestro conocimiento, sólo se han publicado valores normales de aminoácidos en líquidos biologicos mediante cromatografía en papel ${ }^{B}$. método actualmnente insuficiente en el manejo de las aminoacidopatias, según nuestra opinión.

Estu hu motivado la realización de este estudio, cuyo objetivo principal fue establecer valores normales de aminoácidos séricos y urinarios, en una población de niños sanos cuyas edades fluctuaron entre recién nacidos y 6 años de vida.

\section{MATERIAL Y METODO}

Se soleccionaror 108 niños niños sanos de atmbus sexos. con consentimiento informado de sus pedres, cuyas edades fucturan desde 2 días y 6 años de ada procedentes de jardines infantiles y centros hospitalarios de lis $Y$ Región. Las muestras de 7 nir os que presentaron bacteriuria significativi fueron desechadas. al igual que las de 6 niños que presentaron fiebre hasta dos díts despućs de recolectada la muestra. Jín 50 niños se obruvo muestra de sanure $y$ en utros 45 de orina. Los voluntarios se clasificaton en cinco grupos ctarios Cirupo 1: Kecién nacido a 3 mescs (RN-31ij); Grupo 2: 3 meses a 1 año (3m-la): Grupo 3: 1 aก̃o a 2 años (1a-2a); Grupo 4 años a 4 años (2a-4a). Grupo $5: 4$ años a 6 años (4a-6a). Cada grupo esturo constituido por un número igual de niños. Las muestuas de sangre fueron recolectadas en la mañana en un volumen promedio de 3 m]. [nmediatamente formado el cońgulo, se procedió : su separación, al suero obtenido se centrifugó a $\mathbf{3 0 0 0}$ rem. durante 10 minutos. La fracción sérica se removió do cćlulas y se refrigeró $\left(4^{\circ} \mathrm{C}\right.$ ) hasta ser analizada. Las muestras de orina fresca, fueron sometidas en forma inmediatás a pruebas sualitativas (cloruro férico, Be. nodict, 2,4-dinitwofenilhidrazini, método de sciduria metilmalónica). Otra parte de la" muestra de orina fue refirigerada hasta ser anulizada mediante, cromatografia gas-líquido.

La preparación de las muestras para el análisis cuantitutivo, en suero y en orina, comprendjó wna desproteinización con ácido sultosalicílico en una con- centración de 50 mgiml de suero u orina. La solución se centrifugó durante 10 minutos a $3000 \mathrm{rpm}$. Al sobrenadante se le adicionó ef estándar interno Norleucina y se proccctió a aislar los aminó́cidos a través de una resina de intercambio catiónico (Dowex 50 X8-100). El liquido se recibió en micro ampollas de $3 \mathrm{ml}$. se esterificó el grupo carboxito con una mezcla isobutano-HCL $3,2 \mathrm{~m}$ y lucgo sc aciló el grupo amíno con una mezcla cloruro de metilenoranhidrido heptefluobutírico. Los $\mathrm{N}(\mathrm{O}, \mathrm{S})$ HP'B isobutil ésteres de aminócidos se djsolvieron en acctato dc etilo y mediante ura microjeringa (Iamilton 70 IN) se inyectaron en un cromatóforo (Shimadzu GC-8APF), eluipado con doble detcctor de ionización de Jlama. horno con toble columna (columnas de yidrio de 3 ा $\times 2,6$ m D.L. rellenas con $3 \%$ SE-30 sobre Chrom W HP 80/100 mesh), programador de temperaLura, registrador e integrador (Chromatopac C-RIB). EI análisis por cromatografía gas-líquido de los $\mathrm{N}$ (O.S)-HBF Isobutil ésteres de aminoácidos, se desarrolló durante 25 minutos a una temperatura programada entre $100^{\circ} \mathrm{C}$ y $240^{\circ} \mathrm{C}$, con un delta temperatura constante. I as áreas se obtuvier on directamente desde el integrador Chromatopac C-RiB. La jdentificación preliminar de los picos cromatográricos en suero y orina, so realizó por co-inyección de las muestras biológicas con đerivados determinados de aminoácidos y por comparación con los ticmpos de retención correspondientes a mezclds estándares. Eistos timpos de retención se utilizaron para idencificar cada aminoácido en suero y orina. Para lograr la mayor uniformidad en la ident $j$. ficación de los picus, se determinó además el tiempo de retención relativo (TRR) a Noleucina, para cada aninoácido presente en la mezcla estándar, $y$ estos se compararon con los correspondiente a las muestras de sucro y orina. La potencial presencia de diferencias signuficativas, entre los tiempos de retención de mezclas cstúnditres y muestras biológicas, determinó a través de Iis prueba no paramétrica de rangos con signos de Wilcoxon para datos pareados 9 . lil cálculo de la concentración de cada aminoácido per teneciente a una muestra problema, se realizó de acuerdo a la siguiente fórmula:

$A A=($ Area AAp/Arta Sip) $x$ Area Sist $x$ umol AAst RmR x Area AAst $x$ VI

Donde: "R.MR" corresponde a la respuesta molar relativa de detcrminado aninoácido, definida por:

$$
\text { RMR }=\frac{\text { Area A Ast/umol AAst }}{\text { Area Sist/umol Sist }}
$$

$A A p=$ aminoácido en muestra problema

Sip = estándar interno en muestra problema

AAst = aminoácido en mezcla patrón

SIst = estándar interno en mezcla patrón Vi

$\mathrm{Vi}=$ volumen de muestra antes de el intercambio ió. nico.

La información fuc procesada en el computador IBM 4361 de la Universidad Católica de Valparaíso a través del sistema Minitab UC. ${ }^{10}$. Para la determinación de difurencias significativas entre las variables, se utilizó el sistena Statpack ${ }^{11}$. mediante un microcomputados 
Olivetti M 19 del Instituto de Matemáticas y Física de la Universidad de Valparaíso.

\section{RESULTADOS}

Los picos correspondientes a los diferentes aminoácidos, tanto en suero como en orina. lograron una adecuada separación y se caracterizaron por ser simétricos. La figura 1 registra la separación cromatográfica de los 14 aminoácidos en estudio y el estándar interno Norleucina, para una mezcla que cumplió todas las etapas del analisis cuantitativo.

La prueba no paramétrica de rangos con signos de Wilcoxon para datos no pareados, indicó que los tiempos de retención de mezclas estándares eran estadísticamente iguales a los de orina (valor $\mathrm{p}=\mathrm{I}$ ), no así los de suero (valor $\mathrm{p}=$ 0 ). De igual forma, se aplicó esta prueba a los TRR a Norleucina de cada aminoácido, entre mezclas estándares y muestras biológicas, indicando que eran estadisticamente iguales a orina (valor $\mathrm{p}=0,454$ ) y suero (valor $\mathrm{p}=0,190$ ), utilizándose por tanto estos valores para los cálculos posteriores.

El orden de elución de los diferentes aminoácidos concuerda con los reportados en la literatura $^{12,13}$, y fue el siguiente: alanina, glicina, valina, treonina, leucina, isoleucina, norleucina, prolina, metionina, ácido aspártico, fenilalanina, omitina, ácido glutámico, lisina y tirosina. Los tiempos de retención para los 14 aminọacidos estudiados fluctuaron entre 9,60 minutós (alanina) y 22,81 minutos (tirosina). Para el cálculo de los valores se empleó la respuesta molar relativa a norkeucina, la cual presentó una variación entre 0,526 (valina) y 1,333 (Ac. glutámico). El valor promedio de la concentración sérica, rango y desviación estándar para cada aminoácido en 50 niños sanos, cuyas edades flucfuaron entre RN y 6 años, es presentada en la figura 2 .

Los perfiles séricas mostraron concent raciones elevadas de ácido glutámico, alanina y titosina. Las mayores desviaciones estandares las presentan tirosina $(\mathrm{DE}=422,5)$ y ornitina $(\mathrm{DE}=$ $413,5)$.

La figura 3 relaciona las concentraciones promedio de los aminoácidos séricos entre los diferentes grupos etarios. En esta figura se aprecia que: ornitina, lisina, ácido apártico $y$

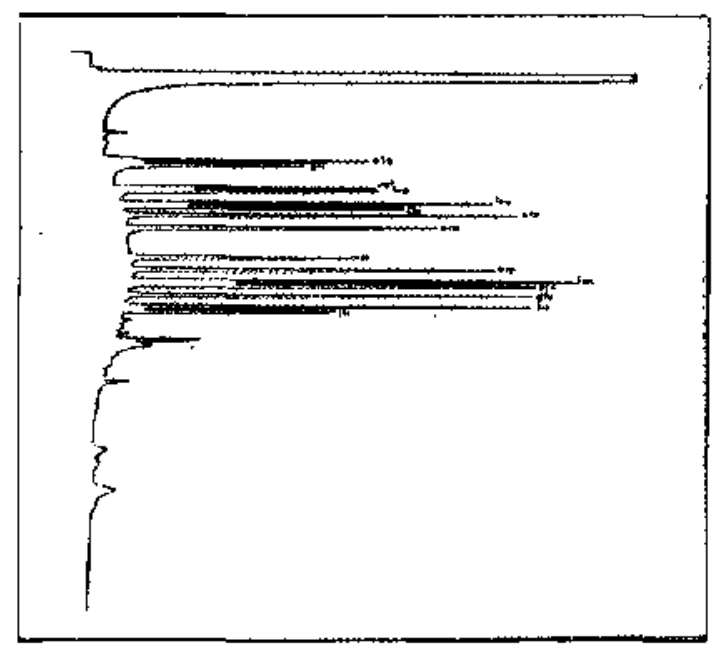

Figura 1: Scparación cromatográlica de $\operatorname{los} \mathrm{N}(0, \mathrm{~S})$-HIःB Isobutil cstéres de aminuácidos en estudio. Columna rellena 3\% S1:-30 (Mezcla estandar de aminoácidos).

$\begin{array}{ll}\text { nle: estándar interno norleucha } \\ \text { ala: alanina } & \text { met: metioniraa } \\ \text { di: glicina } & \text { asp: ac. aspártico } \\ \text { val: valina } & \text { fen. fenilalanina } \\ \text { tre: treonina } & \text { orls: ornitina } \\ \text { leu: 1eucira } & \text { glu: ac. glutámico } \\ \text { jle: isoleucina } & \text { lis: lisina } \\ \text { pro: prolina } & \text { tir: tirosina }\end{array}$

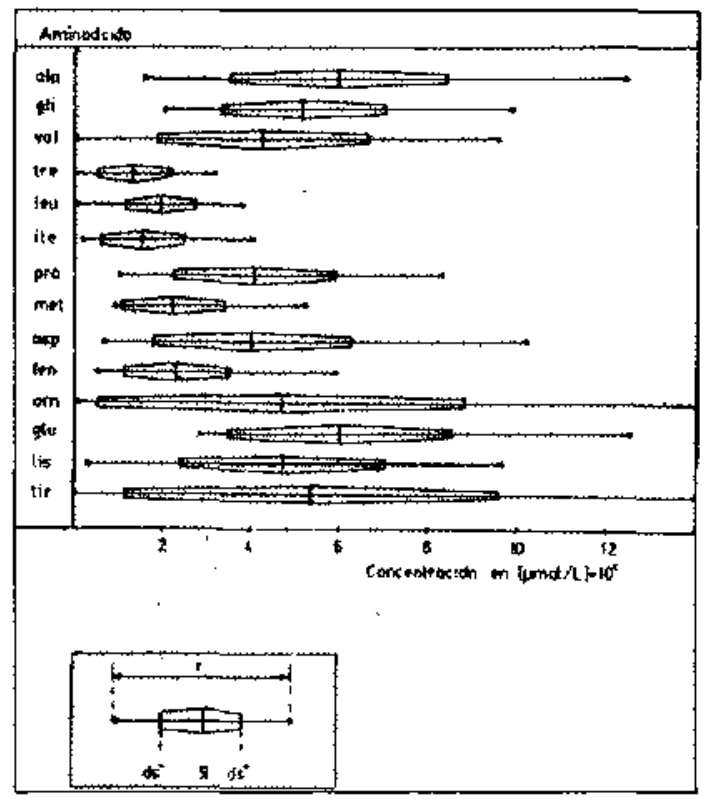

lignua 2: (oncentracionss promedios $(\bar{x})$, rangos (I) y desujaciones estándares (d.s.) de los aminoacidos séricos en 50 niños sanos ( $\mathrm{RN} \cdot 6$ años). 


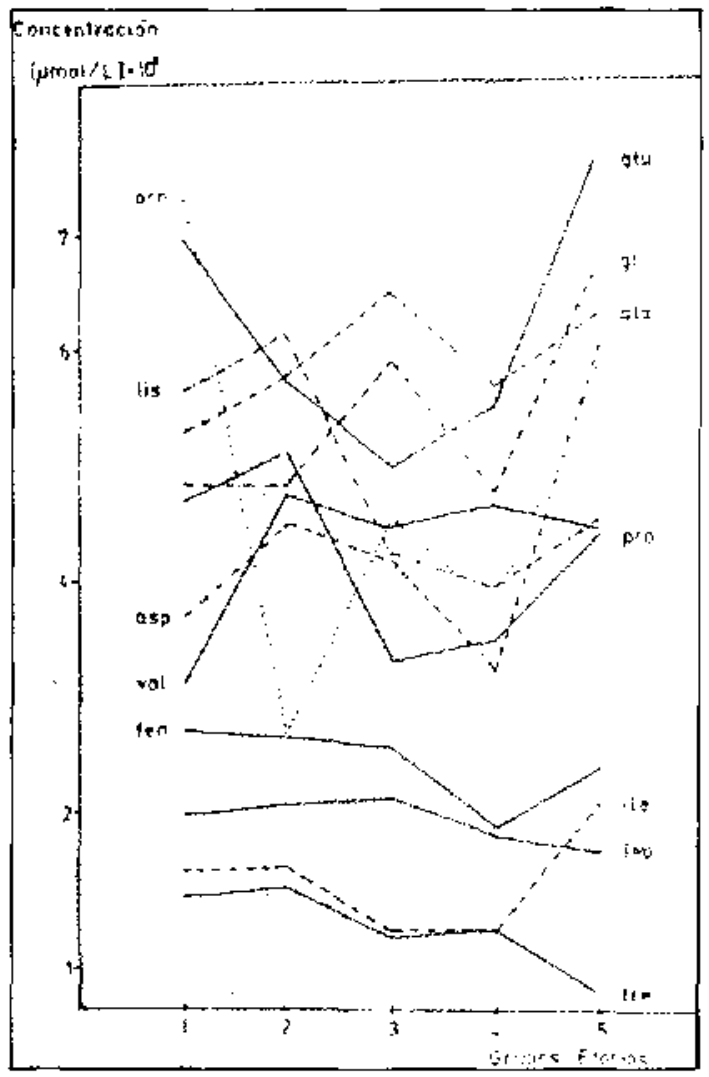

Figura 3: Variacion de la couccentración xicica promedio de 12 aminnácidos un los difterentes gyupos etarion estudiados,

ácido glutámico presentan las mayores fluctuaciones entre los diferentes grupos etarios. Treonina es el aminoácido que presenta la concentración más baja en todos los grupo etarios. Ornitina es el aminoácido que presenta la mayor concentración enl los grupos etarios ( $\mathrm{RN}$ - la). Alanina sc presenta en mayor concentración en los grupos etarios (1a-4a). Acido ylutámico y glutamina se encuentran en mayor concentración en los grupos (RN-3m) y $(4 a-6 a)$. Treonina e isoleucina siguen un comportamiento similar entre los grupos (RN-4a) mientras que en el grupo (4a-6a), los niveles de treonina disminuyen y los de isoleucina aumentan. Metionina $(222 \mathrm{mMol} / 1) \mathrm{y}$ tirosina (539 $\mathrm{mMMol} / 1)$ presentaron promedios prácticamente iguales en los diferentes grupos estudiados, aún cuando la variación dentro de cada grupo etario fue significativa.

La figura 4 presenta las concentraciones promedios, rangos y desviaciones estándar, para

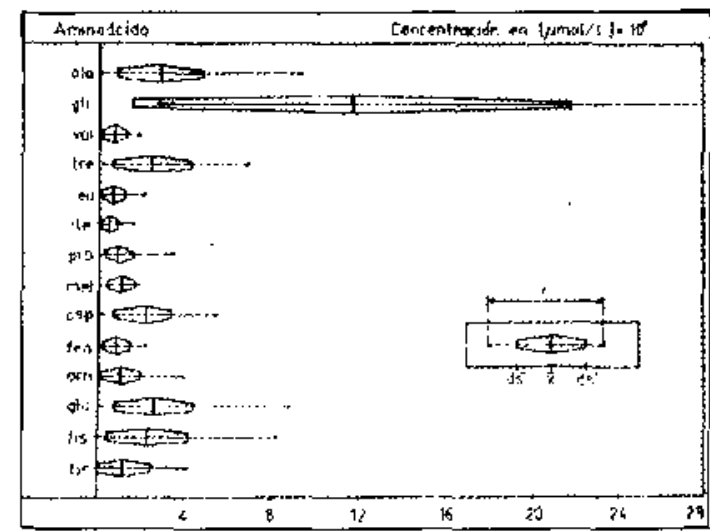

Jigura 4: Conetnuracunes promedios $(\bar{x})$, rangos (c) $y$ desviaciones estindates (d.s.) de los aminódeidos urinarios un 45 niños samos (RN-6 años) expresado en milienoles por lit ro

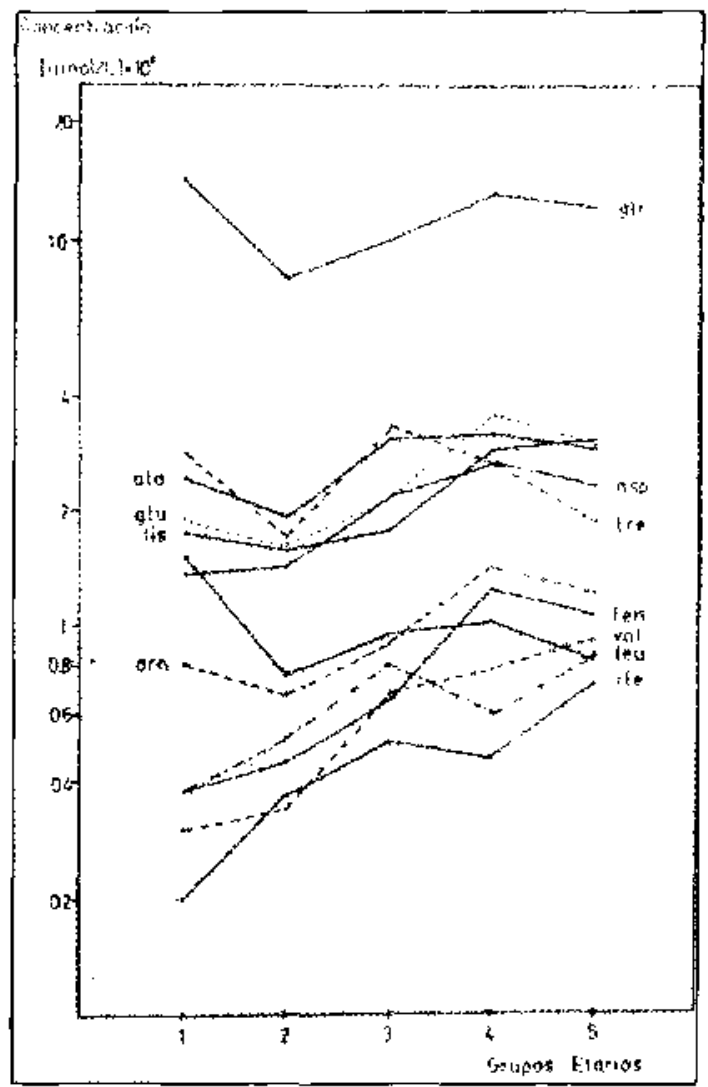

Figura 5: Variación de la concentración urinaria promicdio de 11 ámmócidos en los difcrentes grupos etarios cstudiados.

catorce aminoácidos presentes en orina de 45 niños sanos cuyas cdades fluctuaron entre recién nacidos y seis años. El perfil urinario presentó 
concentraciones elevadas de glicina, aminoácido que posee a su vez, la mayor de las desviaciones eständar $(\mathrm{DE}=1020)$.

$\mathrm{La}$ figura 5 relaciona las concentraciones promedios de los aminoácidos urinarios entre grupos etarios. En la figura se puede observar que: glicina es el aminoácido en mayor concentración en todos los grupos etarios, alanina, treonina, ácido aspártico y lisina, presentan un comportamiento similar entre grupos etarios sin grandes fluctuaciones en sus concentraciones. Isoleucina es el aminoácido en menor concentración en la mayoría de los grupos, a excepción del grupo ( $3 \mathrm{~m}-1 \mathrm{a})$. Fenilalanina presentan su mayor concentración en el grupo (2a-4a), al igual que ornitina. En dicha figura no se presentan tres aminoácidos (prolina, metionina, tirosina), que mostraron un comportamiento promedio muy estable cn los diferentes grupos etarios. El promedio de excreción urinaria de dichos aminoácidos para el total de niños fue de 97,8 mMol/1 para prolina, 110,5 mMol/ 1 para metionina y 123,8 mMol/1 para tirosina.

\section{DISCUSION}

La etapa preliminar de la investigación conprendió el análisis cualitativo de las muestras de orina de todos los pacientes. Dichas pruebas resultaron negativas en todos los casos. Es bien conocido que dichas pruebas pueden dar como resultado falsos negativos o positivos. Frente a la sospecha clínica se deban repetir aquellos que resulten negativos. Si la prueba es positiva, se identificarán y cuantificarán los metabolitos de excreción anormal mediante una técnica de mayor resolución.

La cromatografía gaseosa, frente a otras técnicas cuantitativas empleadas en el laboratorio clínico, presenta la ventaja de una gran versatilidad del instrumento, gran exactitud a concentraciones muy bajas y la posibilidad de combinar con espectrometria de masa para determinar la estructura química de metabolitos no identificados.

En nuestro medio los métodos de cromatografía gaseosa han sido previamente utilizados en el reporte de errores congénitos de metabolismos del grupo de las acidurias orgánicas ${ }^{14}$.

En la interpretación de los datos cuantitativos de aminoácidos, se debe tener presente como posible factor de error, el efecto de venipunturas repetidas, mantención prolongada de la muestra a temperatura ambiente, retraso en la desproteinización de la muestra, contaminación con células sanguíneas y hémolisis ${ }^{15.16}$.

En el caso de la orina, la presencia de enzimas liberadas de tejido renal dafinado o la contaminación con bacterias puede incrementar los aminoácidos por hidrolosis de péptidos y proteínas. Se ha demostrado que la presencia de microorganismos en orina, que contienen hipuricasa, la cual desdobla el ácido hipúrico en ácido benzoico y glicina, eleva los niveles de glicina sobre los normales ${ }^{17}$.

Los niveles de aminoácidos pueden presentar variaciones significativos por procesos metabóli$\cos$ no relacionados con una aminoacidopatia primaria, tales como el estado nutricional, las variaciones circadianas $y$ la infección. Es posible observar diferencias importante en los patrones de excreción de aminoácidos en orina de sujetos sanos, cono consecuencia de hábitos alimentarios. Por otra parte, existen variaciones naturales en la concentración de los aminoácidos según los grupos etarios. En la orina del neonato hay más aminoácidos en base fundamentalmente a glicina. La aminoaciduria denominada fisiológica del prematuro es frecuente $y^{*}$ atribuida a inmadurez renal.

Una anomalía en la excreción de aminoácidos por la orina puede afectar a un aminoácido, a un grupo de los mismos o a todos ellos (Sindrome de Fanconi). Las causas de la excre. ción anormal de un aminoácido son múltiples y varian desde estados catabólicos hasta enfermedades en las cuales existe un déficit especifitico en la reabsorción tubular de algunos aminoácidos (cistinuria). Por tanto cualquier hallazgo inusual en los niveles de aminoácidos urinarios, debe ser confrontado con la clínica y eventualmente decidir una determinación sérica.

Durante el presente trabajo una serie de. muestras correspondientes a enfermedades tales como fenilquetonurias, hiperamonemia por déficit de omitina transcarboxilasa (UTC) ligada al al sexo, acidemia metilmalónica (déficit de succinif $C O A$ transferasa) ${ }^{18}$, e hiperalaninemia, fueron estudiadas en nuestro laboratorio. Debe ser destacado que en todos estos pacientes, algunos de los cuales serán objeto de un posterior reporte, la excreción đe aminoácidos en orina fue significativamente diferente a lo senalado como normal en el presente trabajo. 


\section{RESUMEN}

Los errores congénitos del metabolismo de los aminoácidos se caracterizan por un déficit enzintatico parcial o total, que determina la alteración de una via metabúlica con el consecuente acúmulo de un metabolito. El diag nóstico requiere técnicas de laboratorio exactas confiables, y accesibles.

El objetivo de este trabajo fue desarrollar un método por cromatografía gas-líquido para el análisis de aminoácidos en fluídos biológicos. Las principales etapas del procedimiento experimental fueron aislamiento, derivatización y cuantificación de aminoácidos.

Se establecieron valores normales de aminoácidos en sueto $y$ orina en una población chilena desde recién nacidos a 6 años de edad. Las concentraciones séricas mostraron un am. plio rango de variación entre trazas (tirosina) \ 1808 umol (ornitina). La aminoaciduria nor. mal también presentó una gran variación dependiente de la edad y el tipo de aminoácido en estudio. Estos valores norinales son un clemento necesario en la interpratación de la patologja.

\section{REFERENCIAS}

1. Stanbur J.. Wyndarden J., Fredrickson D., Gold-

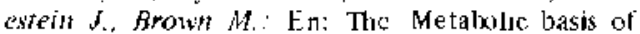
inleried diseass. Me Giaw-Hill Book Co. New York. 3̦til edic. Pág. 7, 1983.

2. Scrimer C $h_{1}$, Rosemberg l., : Lin: Metabolismo de los aminoácidos $y$ sus transtarnos, lidit. Cicntílico Múdica Madrid, pág. 144, 1979.

3. Ciatlure $R$ and Sta A.. A silnple phemylabonine mithod for detection phenylketonurja in large populations ob mewhorn intants. Pedatric 32: 338. 1963

4. Datta S.P. Dent C.E. Marris H.: An apparatus for simultancus production of many twodimensional paper chromatoufams. Science 112: 621, 1950.

5. Moore S., Spackman D.H., Stein W.H.: Chromatography of aminoacids on sulfonated polystyrene resins. Anal Chem 30: 1185, 1958.

6. Darbre $A$, and Istam A.: Gas-liquid cliromatography of trifluoroacetylated amino acid methyl estars Biochem J. 106: 923, 1968.

?. Zumwalt R.W. Kuo $K$, Gehrke C.W'W: Appli cations of a gas-liquid clromatographic method for amino acid analysis. A system for analysis of nanogram amount. J Clromatog 55: 267 , $19 ? 1$.

8. Escobar $C$., Colombo $M$. Jones $K$. Arevalo $L$. Aminoaciduria in población infantil normal de 0 a 6 años. Rey Chil Pediatr 48: 82, 1976.

9. Hollonder $M$ and Wolfe $D$.: Nouparametric statistical methods. Iid. John Wiley \& Sons, New York, pág. 27, 1973.

10. Rodriguez $G$.: Lstadistica descriptiva. En: Computación estadistica interectiva: El sistema MINITABUC. Dpto. listadística, Facultad de Matemáticas, Pontilicia Universidad Católica de Chilc, pág 41. 1986.

1]. Statpack $N_{\text {.: }}$ Single-variable statistic. En: multifunction statistics library. Versión 3.1., Northwest Analytical, Inc, Por thand, Oregon, pág. 9.1, 1984.

12. Mackenzie S. and Tenoschuk D.: Quantitative fotmation of $N(0$, Si-heptalluorobutyril isobutil amino acids for chromatographic analysis: Acylation. J Ciromatogr 173: 53, 1979.

13. Mackenzie $S$, and Tenaschuk D.: Quantitative tormation of $N(0, S)$-heptefluorobutyril isobutyl a mino acids for gas chromatographic analysis: listerification J Chromatogr 173: 53, 1979.

14. Lopez L. Rogrigucz $J$, Bravo P, Bocic J.: Análisis por cromatoprafía gas-líquido de merabolitos urinanos de interés diagnóstico en acidemias orgánicas. Resúmenes de trabajos y conterencias. Primer cncuentro nacional de química analítica Concepción. pág 53, 1987.

15. Soupart $P$.: Free amono acids of blond and urine in the human. in Holden, J. T., ad. Amino Acid Pols. Fiscier, New' York, pás 220, 1962.

16. Rourer G., Jelinek B., Somucts A. J. and Kimugasa $k_{\text {: }}$ Free amino acids in the blood of man and animals Method of study and the effects of venipuncture and food intake on blood free amino acids. In Holden. J.T., \&., Amino Acird Pooks. Llsevier, New York, pp 350, 1962.

17. Malpern B., Pereira N.. Solomon M. Steed E.: A rapld and quantstative gas chromatographic analysis for phenilalavine in serum. Anal Bioch 39: 156, 1971.

18. Garcio A,, Rodriguez J., Pino R.: Acidemia Metilmalónica: a propósito de un caso. Resumen de trabajos. XI Jornadas de la Sociedad Chilena de Pediatría, pág 79, 1985 . 